\title{
SHARP FREQUENCY BOUNDS FOR EIGENFUNCTIONS OF THE ORNSTEIN-UHLENBECK OPERATOR
}

\author{
TOBIAS HOLCK COLDING AND WILLIAM P. MINICOZZI II
}

\begin{abstract}
We prove sharp bounds for the growth rate of eigenfunctions of the OrnsteinUhlenbeck operator and its natural generalizations. The bounds are sharp even up to lower order terms and have important applications to geometric flows.
\end{abstract}

\section{INTRODUCTION}

The Ornstein-Uhlenbeck operator (or drift Laplacian), $\mathcal{L}$ on $\mathbf{R}^{n}$ is the second order operator $\mathcal{L} u=\Delta u-\langle\nabla f, \nabla u\rangle$, where $f=\frac{|x|^{2}}{4}$. It is self-adjoint with respect to the Gaussian $L^{2}$ inner product whose norm is $\|u\|_{L^{2}}^{2}=\int u^{2} \mathrm{e}^{-f}$. We study here the rate of growth of drift eigenfunctions $u$ with $\mathcal{L} u=-\lambda u$. The results given here are important ingredients in the proof of the René Thom gradient conjecture for the arrival time function; see [CM3].

It is easy to see that if $\mathcal{L} u=0$ and $\|u\|_{L^{2}}<\infty$, then $u$ must be constant. More generally, if $\mathcal{L} u=-\lambda u$ and $\|u\|_{L^{2}}<\infty$, then $\lambda$ is a half-integer and $u$ is a polynomial of degree $2 \lambda$. When $n=1$, these polynomials are the Hermite polynomials and the equation $\mathcal{L} u=$ $-\lambda u$ is Hermite's equation. Hermite's equation has a dichotomy where either a solution is polynomial, or it grows faster than any exponential.

We will consider a more general class of drift Schrödinger equations, where $u$ satisfies

$$
\mathcal{L}_{f} u+V u=0,
$$

for some function $V$ and some function $f(x)=f(|x|)$, where $f$ only depends on the distance to the origin. For the Ornstein-Uhlenbeck operator, $f(r)=\frac{r^{2}}{4}$ and, thus, $f^{\prime}(r)=\frac{r}{2}$.

The frequency $U$ of $u$ measures the rate of growth of $u$. If $u(x)=|x|^{d}$, then $U=d$. We show:

Theorem 0.2. Suppose that $f^{\prime}(r) \geq \frac{r}{2}$. Given $\epsilon>0$ and $\delta>0$, there exist $r_{1}>0$ such that if $U\left(\bar{r}_{1}\right) \geq \delta+2 \sup \{0, V\}$ for some $\bar{r}_{1} \geq r_{1}$, then for all $r \geq R\left(\bar{r}_{1}\right)$

$$
U(r)>\frac{r^{2}}{2}-n-2 \sup V-\epsilon .
$$

We will construct examples that show that the lower bound for $U$ is sharp in all dimensions; not only is the quadratic coefficient $\frac{1}{2}$ sharp, but also the constant $-n$ cannot be improved.

The theorem is also sharp in the dependence on the $\sup V$. Namely, if $V=\frac{k}{2}$ is a positive half-integer, then the polynomial solutions mentioned above have $U$ asymptotic to $k$. Thus, the threshold $\delta+2 \sup V$ is sharp. Furthermore, we will see that (0.3) is also $\operatorname{sharp}$ in $\sup V$.

The authors were partially supported by NSF Grants DMS 1404540, DMS 1206827 and DMS 1707270. 
Theorem 0.2 shows that there is a sharp dichotomy for the growth: either $U$ is bounded and $u$ grows at most polynomially, or $u$ grows at least like $r^{-n-2 \sup V} \mathrm{e}^{\frac{r^{2}}{4}}$.

For eigenfunctions of the Ornstein-Uhlenbeck operator, where $f(r)=\frac{r^{2}}{4}$, we also get a lower bound for the derivative of the frequency:

Theorem 0.4. If $f(x)=\frac{|x|^{2}}{4}$ and $\mathcal{L} u+\lambda u=0$, then either $\limsup _{r \rightarrow \infty} U(r) \leq 2|\lambda|$ or there exists $R$ so that for all $r \geq R$

$$
U^{\prime} \geq \frac{r}{2}\left(1+\frac{r^{2}}{2 n+4+4 U(r)-r^{2}}-\frac{2 n+8 \lambda}{2 n+4 U(r)-r^{2}}\right)+\frac{O\left(r^{1-n}\right)}{2 n+4 U(r)-r^{2}},
$$

where $O\left(r^{1-n}\right)$ is a term that is bounded by a constant times $r^{1-n}$.

If we set $W=U-\frac{r^{2}}{4}+\frac{n}{2}$, then (0.5) becomes $W^{\prime} \geq \frac{r}{8}\left(\frac{r^{2}}{W+1}-\frac{2 n+8 \lambda}{W}\right)$ up to lower order terms. Integrating leads to the bound $U \geq \frac{1}{2} r^{2}-n-1-2 \lambda$, which is slightly worse than (0.3). However, this inequality gives a (positive) derivative bound for all values of $U$.

Our arguments are quite flexible and generalize. For instance:

Theorem 0.6. Suppose that $f^{\prime}(r) \geq \frac{r}{2}$. Let $M$ be an open manifold with nonnegative Ricci curvature, Euclidean volume growth and Green's function $G$. Fix $x_{0} \in M$ and let $b$ be given by $b^{2-n}=G\left(x_{0}, \cdot\right)$. Given $\epsilon>0$ and $\delta>0$, there exist $r_{1}>0$ such that if $\mathcal{L}_{f(b)} u=0$ and $U(\bar{r}) \geq \delta$ for some $\bar{r} \geq r_{1}$, then for all $r \geq R(\bar{r})$

$$
U(r)>\frac{r^{2}}{2}-n-\epsilon .
$$

In this theorem, $\mathcal{L}_{f(b)} u=\Delta-\langle\nabla u, \nabla f(b)\rangle$ and $I, D$, and $U$ are defined in terms of $b$; see (3.1), (3.2) and (3.3).

\section{THE SHARP LOWER BOUND FOR $U$}

In this section, $f: \mathbf{R}^{n} \rightarrow \mathbf{R}$ is a function that only depends on the distance to the origin. With slight abuse of notation we write $f(x)=f(|x|)$ and denote $\partial_{r} f$ by $f^{\prime}$.

Define quantities $I(r), D(r)$, and the frequency $U(r)$ by

$$
\begin{aligned}
I(r) & =r^{1-n} \int_{\partial B_{r}} u^{2}, \\
D(r) & =r^{2-n} \int_{\partial B_{r}} u u_{r}=r^{2-n} \mathrm{e}^{f(r)} \int_{B_{r}}\left(|\nabla u|^{2}-V u^{2}\right) \mathrm{e}^{-f}, \\
U(r) & =\frac{D}{I} .
\end{aligned}
$$

The frequency $U$ is the $\log$ arithmic derivative of $\frac{1}{2} \log I$, i.e., $(\log I)^{\prime}=\frac{2 U}{r}$, and thus measures the polynomial rate of growth of $\sqrt{I}$. This frequency was recently used by Bernstein, [B], to study the asymptotic structure of ends of shrinkers for mean curvature flow. It is analogous to a similar quantity for harmonic functions known as Almgren's frequency function, [A], cf. GL], [HS], [L], CM1], [D]. 
An easy calculation together with that $\operatorname{div}\left(\mathrm{e}^{-f} \nabla v\right)=\mathrm{e}^{-f} \mathcal{L}_{f} v$ shows

$$
\frac{d}{d r}\left(r^{1-n} \int_{\partial B_{r}} v\right)=r^{1-n} \int_{\partial B_{r}} \frac{d v}{d r}=r^{1-n}\left(\mathrm{e}^{f(r)} \int_{B_{r}} \mathcal{L}_{f} v \mathrm{e}^{-f}\right) .
$$

Using (1.4) with $\mathcal{L}_{f} u=0$ gives that the spherical average of a $\mathcal{L}_{f}$-harmonic function is constant in $r$.

Lemma 1.5. If $\mathcal{L}_{f} u+V u=0$, then

$$
\begin{aligned}
I^{\prime}(r) & =\frac{2 D(r)}{r}, \\
(\log I)^{\prime}(r) & =\frac{2 U(r)}{r}, \\
D^{\prime}(r) & =\frac{2-n}{r} D+f^{\prime}(r) D+r^{2-n} \int_{\partial B_{r}}\left(|\nabla u|^{2}-V u^{2}\right) .
\end{aligned}
$$

Proof. Since $\mathcal{L}_{f} u^{2}=2|\nabla u|^{2}-2 V u^{2},(1.4)$ gives

$$
I^{\prime}(r)=2 r^{1-n} \mathrm{e}^{f(r)} \int_{B_{r}}\left(|\nabla u|^{2}-V u^{2}\right) \mathrm{e}^{-f}=\frac{2 D(r)}{r} .
$$

This gives the first two claims. Differentiating (1.2) gives (1.8).

Define a (non-linear) first order differential operator on positive functions $g$ on $(0, \infty)$ by

$$
P_{f, \lambda} g=(\log g)^{\prime}+\frac{n-2}{r}-f^{\prime}+\frac{g}{r}+\frac{r \lambda}{g} .
$$

We will later use that if $f_{2}^{\prime} \geq f_{1}^{\prime}$, then $P_{f_{1}, \lambda} g \geq P_{f_{2}, \lambda} g$.

The key will be that $U$ is a sub-solution of $P$ :

Lemma 1.11. If $\mathcal{L}_{f} u+V u=0$ and $\infty>U(r)>0$, then

$$
P_{f, \sup V} U \geq 0 \text {. }
$$

Proof. The Cauchy-Schwarz inequality

$$
\frac{D^{2}}{r}=r^{3-2 n}\left(\int_{\partial B_{r}} u u_{r}\right)^{2} \leq I r^{2-n} \int_{\partial B_{r}} u_{r}^{2} \leq I r^{2-n} \int_{\partial B_{r}}|\nabla u|^{2}
$$

together with (1.8) gives

$$
D^{\prime}(r) \geq \frac{2-n}{r} D+f^{\prime}(r) D+\frac{U}{r} D-r \sup V \frac{D}{U},
$$

Since $(\log U)^{\prime}=\frac{D^{\prime}}{D}-\frac{2 U}{r}$ and $D(r)>0$, dividing (1.14) by $D$ gives (1.12).

The next lemma shows a maximum principle for the operator $P_{f, \lambda}$.

Lemma 1.15. Suppose that $g, h: \mathbf{R} \rightarrow(0, \infty)$ satisfy for $r \geq r_{1}$

$$
P_{f, \lambda} h \geq 0>P_{f, \lambda} g \text {. }
$$

If $h(R)>g(R)$ for some $R \geq r_{1}$, then $h(r)>g(r)$ for all $r \geq R$. 
Moreover, if $\epsilon>0 \geq \lambda$, and $g$ satisfies

$$
-\frac{\epsilon}{r} \geq P_{f, \lambda} g \text { for } r \geq r_{1}
$$

then there exists $R=R\left(h\left(r_{1}\right), g\left(r_{1}\right), r_{1}, \epsilon\right)$ so that $h \geq g$ for $r \geq R$.

Proof. We will prove the first claim by contradiction. Suppose not, then there exists $s>R$ such that $h(s)=g(s)$ and $h(t)>g(t)$ for all $s>t \geq R$. This implies that

$$
(\log h)^{\prime}(s) \leq(\log g)^{\prime}(s)=\frac{g^{\prime}}{g} .
$$

On the other hand, by assumption $P_{f, \lambda} h \geq 0$ and thus

$$
(\log h)^{\prime}(s) \geq \frac{2-n}{s}+f^{\prime}(s)-\frac{h(s)}{s}-s \frac{\lambda}{h(s)}=\frac{2-n}{s}+f^{\prime}(s)-\frac{g(s)}{s}-s \frac{\lambda}{g(s)} .
$$

Together these two inequalities gives that $P_{f, \lambda} g \geq 0$ which is the desired contradiction.

The second claim will follow from the first once we show that there is some $R \geq r_{1}$ so that $h>g$ for some $r$ with $R \geq r \geq r_{1}$. To see this, we suppose that $h \leq g$ for $r_{1} \leq r \leq R$ and then get an upper bound on $R$. On this interval, since $\lambda \leq 0$ we get that

$$
(\log h)^{\prime}(s)-(\log g)^{\prime}(s) \geq P_{f, \lambda} h-P_{f, \lambda} g \geq \frac{\epsilon}{s} .
$$

Integrating this from $r_{1}$ to $R$ gives

$$
1 \geq \frac{h(R)}{g(R)} \geq \frac{h\left(r_{1}\right)}{g\left(r_{1}\right)}\left(\frac{R}{r_{1}}\right)^{\epsilon} .
$$

Thus, we see that $R^{\epsilon} \leq r_{1}^{\epsilon} \frac{g\left(r_{1}\right)}{h\left(r_{1}\right)}$.

Lemma 1.22. Suppose that $f(r)=\frac{r^{2}}{4}, \epsilon>0$ and let $g(r)=\frac{r^{2}}{2}-n-\epsilon-2 \lambda$, then there exists $r_{1}=r_{1}(\epsilon, n)$ so that for $r \geq r_{1}$

$$
-\frac{\epsilon}{2 r} \geq P_{f, \lambda} g
$$

Proof. Choose $r_{1}$ so that for $r \geq r_{1}$

$$
\frac{2(\lambda+1)}{1-2(n+\epsilon+2 \lambda) / r^{2}} \leq 2+2 \lambda+\frac{1}{2} \epsilon .
$$

For $r \geq r_{1},(1.24)$ implies that

$$
\frac{2 r(\lambda+1)}{r^{2}-2(n+\epsilon+2 \lambda)}=\frac{1}{r}\left(\frac{2(\lambda+1)}{1-2(n+\epsilon+2 \lambda) / r^{2}}\right) \leq \frac{2+2 \lambda+\frac{1}{2} \epsilon}{r} .
$$

Using the definitions of $f$ and $g$, we get

$$
-P_{f, \lambda} g=\frac{2-n}{r}+f^{\prime}-\frac{g}{r}-\frac{r \lambda}{g}-\frac{g^{\prime}}{g}=\frac{2+\epsilon+2 \lambda}{r}-\frac{2 r(\lambda+1)}{r^{2}-2(n+\epsilon+2 \lambda)} \geq \frac{\epsilon}{2 r} .
$$

Combining the two previous results and Lemma 1.11 (to see that $P_{f \text {,sup } V} U \geq 0$ ) gives Theorem 0.2 in the case where $\lambda \leq 0$. The argument for a general $\lambda$ is similar but a little more involved since we need a replacement for the second half of Lemma 1.15. We will deal with this in the next subsection. 
1.1. The case $\lambda>0$. The next lemma will replace the second half of Lemma 1.15 when $\lambda>0$.

Lemma 1.27. Suppose that $\lambda>0$ and for $r \geq r_{1}$ we have that $g, h>0, f^{\prime} \geq \frac{r}{2}, P_{f, \lambda} h \geq 0$, $-\frac{\epsilon}{r} \geq P_{f, \lambda} g$, and $r g^{\prime} \geq g$. If $r_{2} \geq r_{1}$ satisfies $\frac{2-n}{r_{2}}+\frac{r_{2}}{2}-r_{2} \frac{\lambda}{\delta+2 \lambda}>\sqrt{\lambda}$ and $h\left(r_{2}\right)>2 \lambda+\delta$, then there exists $R$ such that $h(r) \geq g(r)$ for $r \geq R$.

Proof. First, if $2 \lambda+\delta \leq h(r)<\sqrt{\lambda} r$ for $r \geq r_{2}$, then $P_{f, \lambda} h \geq 0$ implies that

$$
(\log h)^{\prime}(r) \geq \frac{2-n}{r}+\frac{r}{2}-\sqrt{\lambda}-r \frac{\lambda}{\delta+2 \lambda}>0 .
$$

Second, since $P_{f, \lambda} h \geq 0$ and $-\frac{\epsilon}{r} \geq P_{f, \lambda} g$, then

$$
\left(\log \frac{h}{g}\right)^{\prime}=P_{f, \lambda} h-P_{f, \lambda} g+\frac{g-h}{r}-\lambda r \frac{g-h}{g h} \geq \frac{\epsilon}{r}+(g-h)\left(\frac{1}{r}-\frac{\lambda r}{g h}\right) .
$$

Therefore, if $\sqrt{\lambda} r \leq h(r)<g(r)$, then

$$
\left(\log \frac{h}{g}\right)^{\prime}(r) \geq \frac{\epsilon}{r}
$$

and hence, using also that $r g^{\prime} \geq g$ (this is the only place where this is used), we have

$$
(\log h)^{\prime}(r) \geq \frac{\epsilon}{r}+(\log g)^{\prime}(r) \geq \frac{1+\epsilon}{r} .
$$

Thus, when $\sqrt{\lambda} r \leq h(r)<g(r)$, we have that

$$
(h-\sqrt{\lambda} r)^{\prime} \geq \frac{(1+\epsilon) h(r)}{r}-\sqrt{\lambda} \geq(1+\epsilon) \sqrt{\lambda}-\sqrt{\lambda}=\epsilon \sqrt{\lambda}>0 .
$$

In both cases, we get that $h$ only leaves each bound at the upper end and we get an upper bound for the length of the stretch where $h$ has this bound. Finally, it follows from the first part of Lemma 1.15 that once $h$ is above $g$ it stays above.

We can now get rid of the assumption that $r g^{\prime} \geq g$ in Lemma 1.27 to get:

Theorem 1.33. Suppose that $\lambda>0$ and for $r \geq r_{1}$ we have that $g, h>0, f^{\prime} \geq \frac{r}{2}, P_{f, \lambda} h \geq 0$, $-\frac{\epsilon}{r} \geq P_{f, \lambda} g$, then there exists $r_{2}>0$ so that if $h(s)>2 \lambda+\delta$ for some $s \geq r_{2}$, then there exists $R$ so that $h(r) \geq g(r)$ for $r \geq R$.

In particular, $h(r) \geq \frac{r^{2}}{2}-n-2 \lambda-\epsilon$ for $r \geq R$.

Proof. We show the second claim first and then use it to show the first claim. To do that note that if $g_{0}=\frac{r^{2}}{2}-n-2 \lambda-\epsilon$, then $r g_{0}^{\prime}=r^{2} \geq g_{0}$. Moreover, Lemma 1.22 gives $-\frac{\epsilon}{2 r} \geq P_{f, \lambda} g_{0}$. It follows from Lemma 1.27 that for some $R>0$ and all $r>R$ we have that $h(r) \geq \frac{r^{2}}{2}-n-2 \lambda-\epsilon$.

To show the first claim, note that in the proof of Lemma 1.27 the only place where the assumption $r g^{\prime} \geq g$ was used was to show that there exists some $R$ so that once $r \geq R$ and $h(r) \geq \sqrt{\lambda} r$ the function $h$ would stay above the function $\sqrt{\lambda} r$. However, this follows from $h(r) \geq \frac{r^{2}}{2}-n-2 \lambda-\epsilon$ for $r$ large enough.

Proof of Theorem 0.2. We have already proven the case $\lambda \leq 0$. The case $\lambda>0$ follows from Lemma 1.11 and Theorem 1.33 . 
1.2. Sharpness of Theorem $\mathbf{0 . 2}$. The next theorem uses standard solutions of Hermite's equation to show that Theorem 0.2 is sharp even up to the lower order term.

Theorem 1.34. For every $n$ and $k \in \mathbf{Z}$, there is a function $v$ on $\mathbf{R}^{n}$ with $\mathcal{L} v=-\frac{k}{2} v$ whose frequency $U$ goes to infinity but for every $\epsilon>0$ has a sequence $r_{i}$ going to infinity with

$$
U\left(r_{i}\right) \leq \frac{1}{2} r_{i}^{2}-n-k+\epsilon
$$

The second order ODE $\mathcal{L} u=0$ on $\mathbf{R}$, where $f(r)=\frac{r^{2}}{4}$, has a two-parameter family of solutions. The first solution is a constant. The second, $u_{0}(x)$, can be normalized to have $u_{0}(0)=0$ and $u_{0}^{\prime}(0)=1$. The next lemma shows that $I(r) \approx \frac{1}{r} \mathrm{e}^{\frac{r^{2}}{4}}$.

Lemma 1.36. The function $u_{0}$ is odd, $u_{0}^{\prime}(x)=\mathrm{e}^{\frac{x^{2}}{4}}$, and for $x \geq 2$

$$
\mathrm{e}^{\frac{x^{2}}{4}} \leq x u_{0}(x) \leq 6 \mathrm{e}^{\frac{x^{2}}{4}} \text {. }
$$

Moreover, there are functions $u_{k}$ for all $k \in \mathbf{Z}$ with $\mathcal{L} u_{k}=-\frac{k}{2} u_{k}$, so that $u_{k}^{\prime}=u_{k-1}$ and, furthermore, there are constants $c_{k}$ so that

$$
|u(x)| \leq c_{k}|x|^{-k-1} \mathrm{e}^{\frac{x^{2}}{4}} \text { for } 1 \leq|x| .
$$

Proof. Since $\mathcal{L} u_{0}=u_{0}^{\prime \prime}-\frac{x}{2} u_{0}^{\prime}=\mathrm{e}^{\frac{x^{2}}{4}}\left(u_{0}^{\prime} \mathrm{e}^{-\frac{x^{2}}{4}}\right)^{\prime}$, we see that $\left(u_{0}^{\prime} \mathrm{e}^{-\frac{x^{2}}{4}}\right)$ is constant. Using the normalization $u_{0}^{\prime}(0)=1$, the constant is one. For the lower bound given $r>2$, we have

$$
r u_{0}(r)=r \int_{0}^{r} \mathrm{e}^{\frac{x^{2}}{4}} d x \geq \int_{0}^{r} x \mathrm{e}^{\frac{x^{2}}{4}} d x=\left.2 \mathrm{e}^{\frac{x^{2}}{4}}\right|_{0} ^{r}=2 \mathrm{e}^{\frac{r^{2}}{4}}-2 \geq \mathrm{e}^{\frac{r^{2}}{4}} .
$$

To get the upper bound, we divide the integral into three parts

$$
\begin{aligned}
u_{0}(r) & \leq \int_{0}^{1} \mathrm{e}^{\frac{x^{2}}{4}} d x+\int_{1}^{r / 2} x \mathrm{e}^{\frac{x^{2}}{4}} d x+\frac{2}{r} \int_{r / 2}^{r} x \mathrm{e}^{\frac{x^{2}}{4}} d x \leq \mathrm{e}^{\frac{1}{4}}+2\left(\mathrm{e}^{\frac{r^{2}}{16}}-\mathrm{e}^{\frac{1}{4}}\right)+\frac{4}{r} \mathrm{e}^{\frac{r^{2}}{4}} \\
& \leq 2 \mathrm{e}^{\frac{r^{2}}{16}}+\frac{4}{r} \mathrm{e}^{\frac{r^{2}}{4}} \leq \frac{6}{r} \mathrm{e}^{\frac{r^{2}}{4}}
\end{aligned}
$$

where the last inequality used $r^{-1} \mathrm{e}^{\frac{3 r^{2}}{16}}$ is increasing for $r \geq 2$ and $\mathrm{e}^{\frac{r^{2}}{16}} \leq \frac{1}{r} \mathrm{e}^{\frac{r^{2}}{4}}$ at $r=2$.

We construct the $u_{k}$ 's for $k$ inductively for $k<0$ by defining $u_{k}=u_{k+1}^{\prime}$. Using the bound (1.37) and elliptic estimates on balls of radius $|x|^{-1}$ gives the bound (1.38).

For $k \geq 0$, we inductively define

$$
u_{k+1}(x)=\int_{0}^{x} u_{k}(s) d s+d_{k+1},
$$

where the constant $d_{k+1}$ is chosen to make $\mathcal{L} u_{k+1}=-\frac{k+1}{2} u_{k+1}$. To see that we can choose $d_{k+1}$ so that it satisfies the equation, note that

$$
\begin{aligned}
\left(\mathcal{L} u_{k+1}+\frac{k+1}{2} u_{k+1}\right)^{\prime} & =\left(u_{k}^{\prime}-\frac{x}{2} u_{k}+\frac{k+1}{2} u_{k+1}\right)^{\prime} \\
& =u_{k}^{\prime \prime}-\frac{x}{2} u_{k}^{\prime}-\frac{1}{2} u_{k}+\frac{k+1}{2} u_{k}=0 .
\end{aligned}
$$

Using integration by parts, it is easy to see that $u_{k+1}$ grows one degree slower than $u_{k}$ and, thus, satisfies (1.38). 
Proof of Theorem 1.34. It suffices to construct $v_{k}$ with $\mathcal{L} v_{k}=-\frac{k}{2} v_{k}$ where $v_{k}$ grows at least exponentially and has (for all $x$ sufficiently large)

$$
\left|v_{k}\right| \leq C \mathrm{e}^{\frac{|x|^{2}}{2}}|x|^{-k-n} .
$$

This is because the failure of (1.35) for all $r_{i}$ larger than some fixed $R$ implies $\mathrm{e}^{\frac{|x|^{2}}{2}}|x|^{\epsilon-k-n}$ growth $r \geq R$, contradicting (1.43).

The function $u_{k}$ from Lemma 1.36 satisfies (1.43) for $n=1$. For $n>1$, we set

$$
v_{k}\left(x_{1}, \ldots, x_{n}\right)=u_{k}\left(x_{1}\right) u_{0}\left(x_{2}\right) \ldots u_{0}\left(x_{n}\right) .
$$

\section{LOWER BOUND FOR $U^{\prime}$}

In this section, we specialize to the Ornstein-Uhlenbeck operator $\mathcal{L}$ where $f(r)=\frac{r^{2}}{4}$. In this case, $\mathcal{L} f=\frac{n}{2}-f,|\nabla f|^{2}=f$, and the Hessian of $f$ is diagonal with $f_{i j}=\frac{1}{2} \delta_{i j}$.

The next lemma is a drift version of the classical Rellich identity that is used to prove monotonicity of Almgren's frequency for harmonic functions.

Lemma 2.1. Suppose that $\mathcal{L} u+V u=0$ on $\mathbf{R}^{n}$. Given $r>0$, we have

$2 r \int_{\partial B_{r}} u_{r}^{2}-r \int_{\partial B_{r}}\left(|\nabla u|^{2}-V u^{2}\right)=(2-n) \mathrm{e}^{\frac{r^{2}}{4}} \int_{B_{r}}|\nabla u|^{2} \mathrm{e}^{-f}+2 \mathrm{e}^{\frac{r^{2}}{4}} \int_{B_{r}}|\nabla u|^{2} f \mathrm{e}^{-f}$

$$
+2 \mathrm{e}^{\frac{r^{2}}{4}} \int_{B_{r}} V u^{2}\left(\frac{n}{2}-f\right) \mathrm{e}^{-f}+2 \mathrm{e}^{\frac{r^{2}}{4}} \int_{B_{r}} u^{2}\langle\nabla V, \nabla f\rangle \mathrm{e}^{-f} .
$$

Proof. Using that $f_{i j}=\frac{1}{2} \delta_{i j}$, the divergence of $\langle\nabla f, \nabla u\rangle \nabla u-\frac{1}{2}|\nabla u|^{2} \nabla f$ is

$$
\begin{aligned}
\left(f_{i} u_{i} u_{j}-\frac{1}{2} u_{i}^{2} f_{j}\right)_{j} & =f_{i} u_{i} u_{j j}+f_{i} u_{i j} u_{j}+f_{i j} u_{i} u_{j}-\frac{1}{2} u_{i}^{2} f_{j j}-u_{i j} u_{i} f_{j} \\
& =f_{i} u_{i} u_{j j}+\frac{2-n}{4} u_{i}^{2} .
\end{aligned}
$$

In particular, since $\operatorname{div}_{f} X \equiv \mathrm{e}^{f} \operatorname{div}\left(\mathrm{e}^{-f} X\right)=\operatorname{div} X-\langle\nabla f, X\rangle$, we see that

$$
\operatorname{div}_{f}\left(\langle\nabla f, \nabla u\rangle \nabla u-\frac{1}{2}|\nabla u|^{2} \nabla f\right)=\langle\nabla f, \nabla u\rangle \mathcal{L} u+\frac{2-n}{4}|\nabla u|^{2}+\frac{1}{2}|\nabla u|^{2} f,
$$

where the equality also used that $|\nabla f|^{2}=f$. The divergence theorem gives

$$
\begin{aligned}
2 r \int_{\partial B_{r}} u_{r}^{2}-r \int_{\partial B_{r}}|\nabla u|^{2} & =(2-n) \mathrm{e}^{\frac{r^{2}}{4}} \int_{B_{r}}|\nabla u|^{2} \mathrm{e}^{-f}+2 \mathrm{e}^{\frac{r^{2}}{4}} \int_{B_{r}}|\nabla u|^{2} f \mathrm{e}^{-f} \\
& +4 \mathrm{e}^{\frac{r^{2}}{4}} \int_{B_{r}}\langle\nabla f, \nabla u\rangle \mathcal{L} u \mathrm{e}^{-f} .
\end{aligned}
$$

The lemma follows from this and taking $\operatorname{div}_{f}$ of $\frac{1}{2} V u^{2} \nabla f$ to get

$\int_{B_{r}}\langle\nabla f, \nabla u\rangle \mathcal{L} u \mathrm{e}^{-f}=-\frac{1}{2} \int_{B_{r}} V\left\langle\nabla f, \nabla u^{2}\right\rangle \mathrm{e}^{-f}$

$$
=\frac{1}{2} \int_{B_{r}} V u^{2}\left(\frac{n}{2}-f\right) \mathrm{e}^{-f}-\frac{r}{4} \mathrm{e}^{-\frac{r^{2}}{4}} \int_{\partial B_{r}} V u^{2}+\frac{1}{2} \int_{B_{r}} u^{2}\langle\nabla V, \nabla f\rangle \mathrm{e}^{-f} .
$$


We specialize next to drift eigenfunctions, i.e., where $V=\lambda$ is constant.

Lemma 2.6. If $\mathcal{L} u=-\lambda u$ on $\mathbf{R}^{n}$, then

$$
D^{\prime}(r) \geq \frac{r}{2} D+2 \frac{U D}{r}-2 \mathrm{e}^{\frac{r^{2}}{4}} r^{1-n} \int_{B_{r}}\left(|\nabla u|^{2}-\lambda u^{2}\right) f \mathrm{e}^{-f}-2 \lambda \mathrm{e}^{\frac{r^{2}}{4}} r^{1-n} \int_{B_{r}} u^{2} \mathrm{e}^{-f}
$$

Proof. Multiplying Lemma 2.1 by $r^{1-n}$ gives that

$$
\begin{aligned}
2 r^{2-n} \int_{\partial B_{r}} u_{r}^{2}-r^{2-n} \int_{\partial B_{r}}\left(|\nabla u|^{2}-\lambda u^{2}\right) & =(2-n) \frac{D}{r}+2 \mathrm{e}^{\frac{r^{2}}{4}} r^{1-n} \int_{B_{r}}\left(|\nabla u|^{2}-\lambda u^{2}\right) f \mathrm{e}^{-f} \\
& +2 \lambda \mathrm{e}^{\frac{r^{2}}{4}} r^{1-n} \int_{B_{r}} u^{2} \mathrm{e}^{-f} .
\end{aligned}
$$

Using this in the formula for $D^{\prime}$ from Lemma 1.5 gives

$$
\begin{aligned}
D^{\prime}(r) & =\frac{2-n}{r} D+\frac{r}{2} D+r^{2-n} \int_{\partial B_{r}}\left(|\nabla u|^{2}-\lambda u^{2}\right) \\
(2.9) & =\frac{r}{2} D+2 r^{2-n} \int_{\partial B_{r}} u_{r}^{2}-2 \mathrm{e}^{\frac{r^{2}}{4}} r^{1-n} \int_{B_{r}}\left(|\nabla u|^{2}-\lambda u^{2}\right) f \mathrm{e}^{-f}-2 \lambda \mathrm{e}^{\frac{r^{2}}{4}} r^{1-n} \int_{B_{r}} u^{2} \mathrm{e}^{-f} .
\end{aligned}
$$

The lemma follows from this since $r^{2-n} \int_{\partial B_{r}} u_{r}^{2} \geq \frac{U D}{r}$ by (1.13).

The next corollary shows that $U$ is monotone for drift-harmonic functions.

Corollary 2.10. If $\mathcal{L} u=0$ on $\mathbf{R}^{n}$, then

$$
(\log U)^{\prime} \geq \frac{2 \int_{B_{r}}|\nabla u|^{2}\left(\frac{r^{2}}{4}-f\right) \mathrm{e}^{-f}}{r \int_{B_{r}}|\nabla u|^{2} \mathrm{e}^{-f}} \geq 0 .
$$

Proof. Dividing by $D$ in (2.7) with $\lambda=0$, we see that

$$
(\log U)^{\prime} \geq \frac{r}{2}-\frac{2 \int_{B_{r}}|\nabla u|^{2} f \mathrm{e}^{-f}}{r \int_{B_{r}}|\nabla u|^{2} \mathrm{e}^{-f}}=\frac{2 \int_{B_{r}}|\nabla u|^{2}\left(\frac{r^{2}}{4}-f\right) \mathrm{e}^{-f}}{r \int_{B_{r}}|\nabla u|^{2} \mathrm{e}^{-f}} \geq 0 .
$$

When $u$ is not drift harmonic, then we will need to rewrite the right hand side of equation (2.7). This is done next (we record the result for a general $V$ ).

Lemma 2.13. If $\mathcal{L} u+V u=0$ on $\mathbf{R}^{n}$, then

$$
\mathrm{e}^{\frac{r^{2}}{4}} r^{1-n} \int_{B_{r}}\left(|\nabla u|^{2}-V u^{2}\right) f \mathrm{e}^{-f}=\frac{r}{4}(D-I)+\frac{1}{2} \mathrm{e}^{\frac{r^{2}}{4}} r^{1-n} \int_{B_{r}} u^{2}\left(\frac{n}{2}-f\right) \mathrm{e}^{-f} .
$$

Proof. Observe first that since $\mathcal{L} u=-V u, \frac{r^{3-n}}{4} \int_{\partial B_{r}} u u_{r}=\frac{r}{4} D$, and

$$
\operatorname{div}_{f}(u f \nabla u)=\left(|\nabla u|^{2}-V u^{2}\right) f+u\langle\nabla u, \nabla f\rangle,
$$

we have

$$
\mathrm{e}^{\frac{r^{2}}{4}} r^{1-n} \int_{B_{r}}\left(|\nabla u|^{2}-V u^{2}\right) f \mathrm{e}^{-f}=\frac{r}{4} D-\mathrm{e}^{\frac{r^{2}}{4}} r^{1-n} \int_{B_{r}} u\langle\nabla u, \nabla f\rangle \mathrm{e}^{-f} .
$$


Next since $\operatorname{div}_{f}\left(u^{2} \nabla f\right)=2 u\langle\nabla u, \nabla f\rangle+u^{2} \mathcal{L} f=2 u\langle\nabla u, \nabla f\rangle+u^{2}\left(\frac{n}{2}-f\right)$, we have

$$
\mathrm{e}^{\frac{r^{2}}{4}} r^{1-n} \int_{B_{r}} u\langle\nabla u, \nabla f\rangle \mathrm{e}^{-f}=\frac{r}{4} I-\frac{1}{2} \mathrm{e}^{\frac{r^{2}}{4}} r^{1-n} \int_{B_{r}} u^{2}\left(\frac{n}{2}-f\right) \mathrm{e}^{-f} .
$$

Combining these two equations gives the claim.

As a corollary, we get a lower bound for $U^{\prime}$.

Corollary 2.18. If $\mathcal{L} u+\lambda u=0$ on $\mathbf{R}^{n}$, then

$$
U^{\prime}(r) \geq \frac{r}{2}+I^{-1}(r) \mathrm{e}^{\frac{r^{2}}{4}} r^{1-n} \int_{B_{r}} u^{2}\left(f-\frac{n}{2}-2 \lambda\right) \mathrm{e}^{-f} .
$$

Furthermore, given $\delta>0$, there exists $r_{1}$ so that if $U(\bar{r}) \geq \delta+2|\lambda|$ for some $\bar{r} \geq r_{1}$, then there exists $R$ so that for all $r \geq R$

$$
U^{\prime}(r) \geq \frac{r}{2}
$$

Proof. Combining Lemmas 2.6 and 2.13 gives

$$
D^{\prime}(r) \geq 2 \frac{D U}{r}+\frac{r}{2} I+\mathrm{e}^{\frac{r^{2}}{4}} r^{1-n} \int_{B_{r}} u^{2}\left(f-\frac{n}{2}-2 \lambda\right) \mathrm{e}^{-f} .
$$

The first claim follows from this since $U^{\prime}=\left(\frac{D^{\prime}}{D}-\frac{2 U}{r}\right) U$.

To prove the second claim, we just need to show that there is some $R^{2} \geq 2 n+8 \lambda$ with

$$
\int_{B_{R}} u^{2}\left(f-\frac{n}{2}-2 \lambda\right) \mathrm{e}^{-f} \geq 0 .
$$

This follows immediately since $I(r) \mathrm{e}^{-\frac{r^{2}}{4}}$ grows rapidly by Theorem 0.2 .

Proof. (of Theorem 0.4.) We can assume that $\lim _{\sup } \sup _{r \rightarrow \infty} U(r)>2|\lambda|$. Thus, the second part of Corollary 2.18 applies and $U^{\prime}(t) \geq \frac{t}{2}$ for $t>r_{0}$. In particular, $W(t)=U(t)-\frac{t^{2}}{4}$ satisfies $W^{\prime} \geq 0$ for $t \geq r_{0}$. After possibly increasing $r_{0}$, we can assume that $r_{0}^{2}>2 n+8 \lambda$ and, moreover, that $W\left(r_{0}\right)>0$ (using Theorem 0.2).

By Lemma 1.5, for $r>s>r_{0}$

$$
\log \frac{I(s)}{I(r)}=-2 \int_{s}^{r} \frac{U}{t} d t \geq \frac{s^{2}-r^{2}}{4}-2 W(r) \int_{s}^{r} \frac{1}{t} d t=\frac{s^{2}-r^{2}}{4} \log \left(\frac{s}{r}\right)^{2 W(r)}
$$

It follows that for any constant $c \leq r_{0}^{2}$

$$
\begin{gathered}
\mathrm{e}^{\frac{r^{2}}{4}} \frac{r^{1-n}}{I(r)} \int_{r_{0}}^{r}\left(s^{2}-c\right) s^{n-1} I(s) \mathrm{e}^{-\frac{s^{2}}{4}} d s \geq r^{1-n-2 W(r)} \int_{r_{0}}^{r}\left(s^{n+1+2 W(r)}-c s^{n-1+2 W(r)}\right) d s \\
=\frac{r^{3}}{n+2+2 W(r)}-\frac{c r}{n+2 W(r)}+\frac{1}{n+2+2 W(r)} O\left(r^{1-n-2 W(r)}\right),
\end{gathered}
$$

where $O\left(r^{1-n-2 W(r)}\right)$ is a term that is bounded by a constant (depending on $r_{0}$ ) times $r^{1-n-2 W(r)}$. Inserting this in Corollary 2.18 with $c=2 n+8 \lambda$ gives the claim. 


\section{DRIFT HARMONIC FUNCTIONS ON OPEN MANIFOLDS}

In this section, we will show a natural generalization (Theorem 0.6) of (0.3) to open manifolds with nonnegative Ricci curvature and Euclidean volume growth. In fact, the assumptions on the Ricci curvature and volume growth are only used to show that the function $b$ defined below is proper.

Let again $f$ be a function on $(0, \infty)$ with $f^{\prime} \geq \frac{r}{2}$. Suppose that $M$ is an open manifold, $b: M \rightarrow \mathbf{R}$ is a proper function. For a function $u: M \rightarrow \mathbf{R}$, define (cf. [CM1] and [CM2])

$$
\begin{aligned}
I(r) & =r^{1-n} \int_{b=r} u^{2}|\nabla b|, \\
D(r) & =r^{2-n} \mathrm{e}^{f(r)} \int_{b \leq r}|\nabla u|^{2} \mathrm{e}^{-f(b)}, \\
U(r) & =\frac{D(r)}{I(r)} .
\end{aligned}
$$

We set $\mathcal{L}_{f} u=\Delta u-\langle\nabla u, \nabla f(b)\rangle$. It follows that

$$
\begin{aligned}
I^{\prime}(r) & =r^{1-n} \int_{b=r} \frac{\nabla b}{|\nabla b|} u^{2}+\int_{b=r} u^{2} \frac{\nabla b}{|\nabla b|^{2}}\left(r^{1-n}|\nabla b| d \mathrm{Vol}\right) \\
& =r^{1-n} \mathrm{e}^{f(r)} \int_{b \leq r} \mathcal{L}_{f} u^{2} \mathrm{e}^{-f(b)}+\int_{b=r} u^{2} \frac{\nabla b}{|\nabla b|^{2}}\left(r^{1-n}|\nabla b| d \mathrm{Vol}\right),
\end{aligned}
$$

where $d \mathrm{Vol}$ is the volume element of the level set of $b$. The co-area formula gives

$$
D^{\prime}(r)=\frac{2-n}{r} D+f^{\prime}(r) D+r^{2-n} \int_{b=r} \frac{|\nabla u|^{2}}{|\nabla b|} .
$$

If $\mathcal{L}_{f} u=0$, then $\mathcal{L}_{f} u^{2}=2|\nabla u|^{2}$. Therefore

$$
D(r)=\frac{1}{2} r^{2-n} \mathrm{e}^{f(r)} \int_{b \leq r} \mathcal{L}_{f} u^{2} \mathrm{e}^{-f(b)}=r^{2-n} \int_{b=r} u\left\langle\nabla u, \frac{\nabla b}{|\nabla b|}\right\rangle .
$$

The Cauchy-Schwarz inequality (cf. (1.13) $)$ gives for $\mathcal{L}_{f} u=0$

$$
\frac{D^{2}}{r}=r^{3-2 n}\left(\int_{b=r} u u_{r}\right)^{2} \leq I r^{2-n} \int_{b=r} \frac{|\nabla u|^{2}}{|\nabla b|} .
$$

It follows that for $\mathcal{L}_{f} u=0$

$$
D^{\prime}(r)=\frac{2-n}{r} D+f^{\prime} D+r^{2-n} \int_{b=r} \frac{|\nabla u|^{2}}{|\nabla b|} \geq \frac{2-n}{r} D+f^{\prime} D+\frac{U}{r} D .
$$

If $\mathcal{L}_{f} u=0$ and

$$
\frac{\nabla b}{|\nabla b|^{2}}\left(r^{1-n}|\nabla b| d \mathrm{Vol}\right)=0
$$

then $I^{\prime}=2 \frac{D}{r}$ and $(\log I)^{\prime}=\frac{2 U}{r}$. Hence, by (3.8)

$$
P_{f, 0} U \geq 0 \text {. }
$$

By [CM1] if $b^{2-n}$ is harmonic, then (3.9) holds. This is due to the following: 
Lemma 3.11. Let $d \mathrm{Vol}$ denote the volume element of the level set of a function $v$, then

$$
\frac{\nabla v}{|\nabla v|^{2}}(|\nabla v| d \mathrm{Vol})=\frac{\Delta v}{|\nabla v|} d \mathrm{Vol} \text {. }
$$

Proof. An easy calculation shows that the change in volume element (of the level set) is

$$
\operatorname{div}\left(\frac{\nabla v}{|\nabla v|^{2}}\right)-\left\langle\nabla_{\frac{\nabla v}{|\nabla v|}}\left(\frac{\nabla v}{|\nabla v|^{2}}\right), \frac{\nabla v}{|\nabla v|}\right\rangle=\frac{\Delta v}{|\nabla v|^{2}}-\frac{\nabla v(|\nabla v|)}{|\nabla v|^{3}} .
$$

From this the claim follows.

It follows from (3.10) together with Theorem 1.33 that:

Theorem 3.14. Suppose that $f^{\prime} \geq \frac{r}{2}$. Given $\epsilon>0$ and $\delta>0$, if $\Delta b^{2-n}=0$, then there exist $r_{1}>0$ so that if $U(\bar{r}) \geq \delta$ for some $\bar{r} \geq r_{1}$ and $\mathcal{L}_{f} u=0$, then for all $r \geq R(\bar{r})$

$$
U(r)>\frac{1}{2} r^{2}-n-\epsilon .
$$

In particular, it follows from [CM1] that if $M$ is an open manifold with nonnegative Ricci curvature and Euclidean volume growth and $b$ is given by $b^{2-n}=G$, then $b$ is proper and thus the conclusion of Theorem 3.14 holds giving Theorem 0.6. (Here $G$ is the Green's function.)

\section{Approximation of eigenfunctions}

Theorem 0.2 implies that if $\mathcal{L} u=-\lambda u$ on $\mathbf{R}^{n}$, then either $u$ grows at most polynomially or at least as fast at $r^{-p} \mathrm{e}^{\frac{r^{2}}{4}}$ for some power $p$. In the first case, $\|u\|_{L^{2}}<\infty$, so $u$ is a polynomial and $\lambda$ a half-integer. The next theorem gives a local version of this; we will see a more general version of this in the next section.

Theorem 4.1. Given $k \in \mathbf{Z}$ and $R_{0}$, there exist $C$ and $R_{1}$ so that if $\mathcal{L} u=-\frac{k}{2}$ on $B_{R}$ for some $R \geq R_{1}$, then there is a polynomial $v$ of degree at most $k$ so that

$$
\sup _{B_{R_{0}}}|u-v|^{2} \leq C R^{4 n-1+\max \{0,2 k+2\}} \mathrm{e}^{-\frac{R^{2}}{2}} \int_{B_{R+\frac{1}{R}} \backslash B_{R-\frac{1}{R}}} u^{2} .
$$

Proof. We will prove this in two steps. Suppose first that $k \leq-1$. Lemma 1.11 gives

$$
(\log U)^{\prime} \geq \frac{2-n}{r}+\frac{r}{2}+\frac{r}{2 U}-\frac{U}{r} .
$$

We will show first that $U$ goes above $n$ on any interval $\left[r_{0}, r_{0}+1\right]$ for $r_{0} \geq 2 n$. To see this, suppose that $U \leq n$ on such an interval and use (4.3) to get that

$$
U^{\prime} \geq \frac{r}{2}+U\left(\frac{2-n}{r}+\frac{r}{2}-\frac{n}{r}\right)>n .
$$

This is impossible since $0 \leq U \leq n$, giving the claim. Thus, Theorem 0.2 gives $\bar{R}$ depending on $n$ so that $U(r)>\frac{r^{2}}{2}-n$ for all $r \geq \bar{R}$. Given $r \geq \bar{R}$, integrating this from $r$ to $R$ gives

$$
\log \frac{I(R)}{I(r)} \geq 2 \int_{r}^{R}\left(\frac{s}{2}-\frac{n}{s}\right) d s=\frac{1}{2}\left(R^{2}-r^{2}\right)-2 n \log \frac{R}{r} .
$$


Letting $r=\min \left\{\bar{R}, 2 R_{0}\right\}$, exponentiating and applying elliptic estimates gives

$$
\sup _{B_{R_{0}}}|u|^{2} \leq c I(r) \leq C R^{2 n} \mathrm{e}^{-\frac{R^{2}}{2}} I(R) .
$$

The case $k \leq-1$ follows from this since $I(R) \leq c R^{2-n} \int_{B_{R+\frac{1}{R}} \backslash B_{R}} u^{2}$.

Suppose now that $k \geq 0$ and let $w$ be any $(k+1)$-st partial derivative of $u$. It follows that $\mathcal{L} w=-\frac{1}{2} w$, so (4.6) implies that

$$
\sup _{B_{R_{0}}}\left|\nabla^{k+1} u\right|^{2} \leq C R^{2 n} \mathrm{e}^{-\frac{R^{2}}{2}} \int_{\partial B_{R}}\left|\nabla^{k+1} u\right|^{2} .
$$

Elliptic estimates on balls of radius $R^{-1}$ centered on $\partial B_{R}$ give that

$$
\sup _{\partial B_{R}}\left|\nabla^{k+1} u\right|^{2} \leq C R^{2 k+2+n} \int_{B_{R+\frac{1}{R}} \backslash B_{R-\frac{1}{R}}} u^{2}
$$

The theorem follows with $v$ given by the degree $k$ Taylor polynomial for $u$ at 0 .

\section{Approximate eigenfunctions on CYlinders}

In this section, we let $M=N \times \mathbf{R}^{n}$ be a product manifold where $N$ is closed. Let $x$ be coordinates on $\mathbf{R}^{n}$, define $f=\frac{|x|^{2}}{4}$ and the drift Laplacian $\mathcal{L}=\Delta-\frac{1}{2} \nabla_{x}=\Delta_{N}+\mathcal{L}_{\mathbf{R}^{n}}$. Given a function $u$, we define $I$ and $D$ by

$$
\begin{aligned}
I(r) & =r^{1-n} \int_{|x|=r} u^{2}, \\
D(r) & =r^{2-n} \int_{|x|=r} u u_{r}=\mathrm{e}^{\frac{r^{2}}{4}} r^{2-n} \int_{|x|<r}\left(|\nabla u|^{2}+u \mathcal{L} u\right) \mathrm{e}^{-f} .
\end{aligned}
$$

Here $u_{r}$ denotes the normal derivative of $u$ on the level set $|x|=r$. Since $N$ is compact, $f$ is proper and the integrals exist. It is easy to see that $I^{\prime}=\frac{2 D}{r}$ and $(\log I)^{\prime}=\frac{2 U}{r}$, where the frequency $U$ is given by $U=\frac{D}{I}$.

The next theorem gives a strong approximation for approximate eigenfunctions on $M$. The theorem is stated for eigenvalue $-\frac{1}{2}$ for simplicity, but can be modified easily for other eigenvalues by arguing as in the previous section. This result is a key ingredient in [CM3].

Theorem 5.3. There exist $\bar{R}$ and $C$ depending on $n$ so that if $v$ is a function on $\{|x| \leq R\}$, where $\bar{R} \leq R$, and

(1) $\left|-\frac{1}{2} v^{2}+v \mathcal{L} v\right| \leq \psi^{2}+\epsilon\left(\frac{v^{2}}{2}+|\nabla v|^{2}\right)$, where $\psi$ is a function and $\epsilon<\frac{1}{2}$,

then we get for any $\Lambda \in(0,1 / 2)$ that

$$
\int_{|x|<4 n} v^{2} \mathrm{e}^{-f} \leq \frac{2}{\Lambda}\|\psi\|_{L^{2}}^{2}+C I(R) R^{2 n} \mathrm{e}^{-\frac{(1-\epsilon-\Lambda) R^{2}}{2(1+\epsilon+\Lambda)^{2}}}
$$

In the proof, we will need a modified version of the frequency. Define $E(r)$ by

$$
E(r)=r^{2-n} \mathrm{e}^{\frac{r^{2}}{4}} \int_{|x|<r}\left\{|\nabla v|^{2}+\frac{1}{2} v^{2}\right\} \mathrm{e}^{-f}=D(r)-r^{2-n} \mathrm{e}^{\frac{r^{2}}{4}} \int_{|x|<r}\left(v \mathcal{L} v-\frac{1}{2} v^{2}\right) \mathrm{e}^{-f} .
$$


We define a modified frequency $U_{E}$ by

$$
U_{E}(r)=\frac{E(r)}{I(r)}
$$

Lemma 5.6. We have

$$
\left(\log U_{E}\right)^{\prime} \geq \frac{2-n}{r}+\frac{r}{2}+\frac{r}{2 U_{E}}+\frac{U}{r}\left(\frac{D}{E}-2\right) .
$$

Proof. Differentiating gives that

$$
E^{\prime}(r)=\frac{2-n}{r} E+\frac{r}{2} E+\frac{r}{2} I+r^{2-n} \int_{|x|=r}|\nabla v|^{2} \geq \frac{2-n}{r} E+\frac{r}{2} E+\frac{r}{2} I+\frac{U D}{r},
$$

where the inequality used the Cauchy-Schwarz inequality, (1.13). The lemma follows from this since $\frac{I^{\prime}}{I}=\frac{2 U}{r}$.

Proof of Theorem 5.3. We get (5.4) immediately if

$$
\int_{|x|<4 n} v^{2} \mathrm{e}^{-f}<\frac{2}{\Lambda}\|\psi\|_{L^{2}}^{2}
$$

Suppose, therefore, that (5.9) fails. Given any $r \geq 4 n$, it follows from (1) that

$$
|D-E| \leq \epsilon E+r^{2-n} \mathrm{e}^{\frac{r^{2}}{4}}\|\psi\|_{L^{2}}^{2} \leq \epsilon E+\Lambda r^{2-n} \mathrm{e}^{\frac{r^{2}}{4}} \int_{|x|<4 n} \frac{v^{2}}{2} \mathrm{e}^{-f} \leq(\epsilon+\Lambda) E .
$$

Therefore, if $4 n \leq r$, then: $0 \leq I^{\prime}(r)$,

$$
\begin{aligned}
\left|U-U_{E}\right|(r) & \leq(\epsilon+\Lambda) U_{E}(r), \\
\left(\log U_{E}\right)^{\prime} & \geq \frac{2-n}{r}+\frac{r}{2}+\frac{r}{2 U_{E}}-(1+\epsilon+\Lambda)^{2} \frac{U_{E}}{r},
\end{aligned}
$$

where the last inequality also used Lemma 5.6.

We first show that $\max _{[4 n, 8 n]} U_{E} \geq n$. To see this, suppose instead that $U_{E}<n$ on $[4 n, 8 n]$ and use (5.12) to get

$$
\left(\log U_{E}\right)^{\prime}>\frac{2 n}{U_{E}}
$$

Multiplying by $U_{E}$, we get an interval of length $4 n$ where $0<U_{E}<n$ but $2 n<U_{E}^{\prime}$. This is impossible, so we conclude that $\max _{[4 n, 8 n]} U_{E} \geq n$ as claimed.

We claim that there exists $\bar{R}=\bar{R}(n) \geq 5 n$ so that for all $r \geq \bar{R}$ we have

$$
U_{E}(r)>\frac{r^{2}-2 n}{2(1+\epsilon+\Lambda)^{2}} \text {. }
$$

The key is that if (5.14) fails for some $r \geq 4 n$, then (5.12) implies that

$$
\left(\log U_{E}\right)^{\prime} \geq \frac{2}{r}+\frac{r}{2 U_{E}} \geq \frac{6}{r} .
$$

On the other hand, for $r \geq 4 n$, we have

$$
\left(\log \frac{r^{2}-2 n}{2(1+\epsilon+\Lambda)^{2}}\right)^{\prime}=\frac{2 r}{r^{2}-2 n}<\frac{3}{r}
$$


Integrating (5.15) and (5.16) and using that $\max _{[4 n, 8 n]} U_{E} \geq n$, gives an upper bound for the maximal interval where (5.14) fails. The first derivative test, (5.15), and (5.16) imply that once (5.14) holds for some $R \geq 4 n$, then it also holds for all $r \geq R$. This gives the claim.

Using (5.11) and (5.14), we get for $r \geq \bar{R}$ that

$$
U(r) \geq(1-\epsilon-\Lambda) U_{E}(r)>\frac{(1-\epsilon-\Lambda)}{(1+\epsilon+\Lambda)^{2}}\left(\frac{r^{2}}{2}-n\right) \equiv \kappa\left(\frac{r^{2}}{2}-n\right),
$$

where the last equality defines $\kappa$. Integrating this from $\bar{R}$ to $R$ gives that

$$
\log \frac{I(R)}{I(\bar{R})} \geq 2 \int_{\bar{R}}^{R} \frac{U(r)}{r} d r \geq \kappa \int_{\bar{R}}^{R}\left(r-\frac{2 n}{r}\right) d r=\kappa\left(\frac{R^{2}-\bar{R}^{2}}{2}-2 n \log \frac{R}{\bar{R}}\right) .
$$

Since $\bar{R}$ is uniformly bounded, exponentiating gives that

$$
\sup _{4 n \leq r \leq \bar{R}} I(r)=I(\bar{R}) \leq c_{n} I(R) R^{2 n \kappa} \mathrm{e}^{-\frac{\kappa}{2} R^{2}}
$$

We use the reverse Poincaré to get the integral bound on $|x|<4 n$. Let $\eta \leq 1$ be a cutoff that is one on $\{|x|<4 n\}$, zero for $|x|>5 n$, and has $|\nabla \eta| \leq 1$. Integration by parts gives

$$
\int \eta^{2}\left(|\nabla v|^{2}+\frac{v^{2}}{2}\right) \mathrm{e}^{-f}=-\int\left(2 \eta v\langle\nabla v, \nabla \eta\rangle+\eta^{2}\left(v \mathcal{L} v-\frac{v^{2}}{2}\right)\right) \mathrm{e}^{-f} .
$$

Using $(2)$ on the last term (note that $\epsilon<1 / 2$ ) and absorbing the first term gives

$$
\begin{aligned}
\frac{1}{2} \int \eta^{2}\left(|\nabla v|^{2}+\frac{v^{2}}{2}\right) \mathrm{e}^{-f} & =\|\psi\|_{L^{2}}^{2}-\int(2 \eta v\langle\nabla v, \nabla \eta\rangle) \mathrm{e}^{-f} \\
& \leq\|\psi\|_{L^{2}}^{2}+\frac{1}{2} \int \eta^{2} v|\nabla v|^{2} \mathrm{e}^{-f}+2 \int|\nabla \eta|^{2} v^{2} \mathrm{e}^{-f}
\end{aligned}
$$

Since $\eta=1$ for $|x|<4 n$ and $|\nabla \eta| \leq 1$ is only nonzero for $4 n<|x|<5 n$, it follows that

$$
\int_{\{|x|<4 n\}} v^{2} \mathrm{e}^{-f} \leq 4\|\psi\|_{L^{2}}^{2}+8 \int_{\{4 n<|x|<5 n\}} v^{2} \mathrm{e}^{-f} \leq 4\|\psi\|_{L^{2}}^{2}+C I(5 n),
$$

where we used that $I^{\prime}(r) \geq 0$ for $r \geq 4 n$. Combining (5.19) and (5.22) gives (5.4).

\section{REFERENCES}

[A] F. Almgren, Jr., Q-valued functions minimizing Dirichlet's integral and the regularity of area minimizing recti able currents up to codimension two, preprint.

[B] J. Bernstein, Asymptotic structure of almost eigenfunctions of drift Laplacians on conical ends, preprint, https://arxiv.org/pdf/1708.07085.pdf.

[CM1] T.H. Colding and W.P. Minicozzi II, Harmonic functions of polynomial growth, JDG 46 (1997), 1-77.

[CM2] _ Large scale behavior of kernels of Schrödinger operators, American Journal of Mathematics, Vol. 119, No. 6 (1997), 1355-1398.

[CM3],$R$. Thom's gradient conjecture for the arrival time, preprint.

[D] C. De Lellis, The size of the singular set of area-minimizing currents, Surveys in differential geometry 2016. Advances in geometry and mathematical physics, 1-83, Surv. Differ. Geom., 21, Int. Press, Somerville, MA, 2016.

[GL] N. Garofalo and F.H. Lin, Monotonicity properties of variational integrals, $A_{p}$ weights and unique continuation, Indiana Univ. Math. J. 35 (1986), no. 2, 245-268.

[HS] R. Hardt and L. Simon, Nodal sets for solutions of elliptic equations, JDG 30 (1989), 505-522. 
[L] F.H. Lin, Nonlinear theory of defects in nematic liquid crystals; phase transition and flow phenomena, Commu. on Pure and Applied Math. 42 (1989), 789-814.

Mit, Dept. of Math., 77 Massachusetts Avenue, Cambridge, MA 02139-4307.

E-mail address: colding@math.mit.edu and minicozz@math.mit.edu 